Canad. Math. Bull. Vol. 21 (4), 1978

\title{
IDEMPOTENTS IN THE GROUPOID OF ALL SP CLASSES OF LATTICES
}

\author{
BY \\ ALAN DAY ${ }^{(1)}$
}

1. Introduction. In [5], Mal'cev generalized the group theoretical results of H. Neumann (see [6] Chapter 2) to produce the notion of the product, $\mathscr{A} \cdot \mathscr{B}$, of two subclasses of a given variety of algebras, $\mathscr{K}$. Following the group theorectic example, members of $\mathscr{A} \cdot \mathscr{B}$ were called extensions of algebras in $\mathscr{A}$ by algebras in $\mathscr{B}$. When $\mathscr{K}=\mathscr{L}$, the variety of all lattices, this product has been investigated for example by Lender [4] and at the Oberwolfach meeting in 1976, Shevrin posed the following conjecture:

Are $\mathscr{L}$ and $\mathscr{T}$ the only varieties of lattices idempotent under this product? ( $\mathscr{T}$ is the variety of all lattices satisfying $x=y$ )

The purpose of this note is to answer this conjecture affirmatively.

2. Preliminaries. If $\mathscr{A}$ and $\mathscr{B}$ are abstract classes of lattices, their Mal'cev product is defined by: $C \in \mathscr{A} \cdot \mathscr{B}$ iff for some $\theta \in \operatorname{Con}(C), C / \theta \in \mathscr{B}$ and for all $x \in C,[x]_{\theta} \in \mathscr{A}$. $\left([x]_{\theta}\right.$ is the congruence class of $x$ modulo $\theta$.) A prevariety of lattices is a subclass of $\mathscr{L}$ closed under $\mathbf{S}$ and $\mathbf{P}$, and as shown in [5] the Mal'cev product of prevarieties is again such. We should also note that any non-trivial prevariety contains all distributive lattices.

We also need a construction in lattices defined originally in [1]. If $A$ is a lattice and $I=[u, v]$ is a closed interval in $A$, then $A[I]=(A \backslash I) \cup(I \times 2)$ is a lattice with the product order relation on $I \times 2$ and the original (and/or first projection order relation otherwise). There is a natural epimorphism $\kappa_{I}: A[I] \rightarrow \mathscr{A}$. We define Int $\mathscr{A}=\{A[I]: A \in \mathscr{A}$ and $I=[u, v] \leq A\}$.

We also need some facts about free lattices. For $A \in \mathscr{L},(a, b, c, d) \in A^{4}$ satisfies Whitman's condition iff $(W): a \wedge b \leq c \vee d$ implies $\{a, b, c, d\} \cap$ $[a \wedge b, c \vee d] \neq \phi$. This condition comes from the well-known solution to the word problem for free lattices given in Whitman [7]. The form of this theorem needed here is in Jónsson [3].

(2.1) TheOREM. Let $L$ be a lattice generated by a subset $X \subseteq L$; then $L$ is freely generated by $X$ if and only if $L$ satisfies $(W)$ and for all finite subsets $Y, Z \subseteq X$, $\wedge Y \leq \vee Z$ iff $Y \cap Z \neq \phi$.

The following result from [2] is also needed.

(1) This research was supported by the National Research Council.

Received by the editors July 21, 1977 and in revised form, February 13, 1978. 
(2.2) TheOREM. For each lattice $A$ in $\mathscr{L}$ there exists a sequence of lattices $\left(A_{n}\right)_{n \in N}$ and epimorphisms $\rho_{n}: A_{n+1} \rightarrow A_{n}$ such that

$$
A_{0}=A \quad \text { and } \quad A_{n+1} \in \mathbf{S P} \operatorname{Int}\left\{A_{n}\right\}
$$

$$
A_{\infty}=\lim _{\leftarrow}\left(A_{n^{\prime}} \rho_{n}\right) \text { satisfies }(W)
$$

3. The results. While some of the results stated below obviously hold under weaker assumptions we will assume all classes of lattices considered are prevarieties.

(3.1) Lemma. For prevarieties $\mathscr{A}$ and $\mathscr{B}$ with $\mathscr{A}$ non-trivial, Int $\mathscr{B} \subseteq \mathscr{A} \cdot \mathscr{B}$.

Proof. The congruence classes of $\kappa_{I}: B[I] \rightarrow B$ are isomorphic to either 1 or 2 both of which belong to $\mathscr{A}$.

(3.2) Corollary. If $\mathscr{A}$ is a non-trivial prevariety that is idempotent then Int $(\mathscr{A}) \subseteq \mathscr{A}$.

(3.3) TheOREM. Any idempotent non-trivial prevariety $\mathscr{A}$ contains $F L(X)$, the free lattice on $X$ generators, for each set $X$.

Proofs. As $\mathscr{A}$ is non-trivial, we have that for any set $X, F D(X)$, the free distributive lattice on $X$ generators, belongs to $\mathscr{A}$. Now using $A_{0}=F D(X)$ in (2.2) we have by the lemma, $A_{n} \in \mathscr{A}$ for all $n \in N$ and therefore also $A_{\infty} \in \mathscr{A}$. Now if $\rho_{\infty}: A_{\infty} \rightarrow A_{0}=F D(X)$ is the canonical epimorphism, then any set of representatives $\bar{X}$ from $\left\{\rho_{\infty}^{-1}(x): x \in X\right\}$ must satisfy the second property of (2.1). Since we also have $A_{\infty}$ satisfying (W), we have by (2.1), $F L(X) \simeq\langle\bar{X}\rangle \in \mathscr{A}$.

(3.4) Corollary. If $\mathscr{V}$ is a variety of lattices that is idempotent then $\mathscr{V}=\mathscr{T}$ or $\mathscr{V}=\mathscr{L}$.

Proof. If $\mathscr{V} \neq \mathscr{T}$ then since $\mathscr{V}$ is a prevariety, we have by the theorem $F L(X) \in \mathscr{V}$ for all sets $X$. Since $\mathscr{V}$ is also closed under $\mathbf{H}$, this forces $\mathscr{V}=\mathscr{L}$.

Acknowledgement. The author would like to thank Professors Gratzer and Shevrin in particular and the Oberwolfach committee in general; for the discussion that led to this result.

\section{REFERENCES}

1. A. Day, A Simple Solution to the Word Problem for Lattices, Can. Math. Bull. 13 (1970), 253-254.

2. A. Day, Splitting Lattices Generate All Lattices, Proceedings of the Conference on Universal Algebra, Szeged 1975, North-Holland (in print).

3. B. Jónsson, Relatively Free Lattices, Colloq. Math. 21 (1970), 191-196.

4. W. B. Lender, About a Groupoid of Prevarieties of Lattices, Siberian Math. J. XVI No 6 (1975), 1214-1223 (Russian). 
5. A. I. Mal'cev, Multiplication of Classes of Algebraic Systems, Siberian Math J. 8 (1976), 764-770 (Russian). Translation available in: A. I. Mal'cev, The Metamathematics of Algebraic Systems, Studies in Logic Vol. 66, North-Holland, Amsterdam (1971).

6. H. Neumann, Varieties of Groups, Erg. der Math, New Series Vol. 37, Springer-Verlag, Berlin 1967.

7. P. Whitman, Free Lattices, Ann. of Math. 42 (1941), 325-330.

Department of Mathematical Science

LAKEHEAD UNIVERSITY

Thunder BAy, ONT. P7B 5E1 